\title{
Metabolomics approach for multibiomarkers determination to investigate dengue virus infection in human patients
}

\author{
Nurul Shahfiza', Hasnah Osman², Tang Thean Hock and Abdel-Hamid Zaki Abdel-Hamid ${ }^{1,3 凶}$ \\ ${ }^{1}$ Advanced Medical and Dental Institute, University Science Malaysia, Malaysia; ${ }^{2}$ School of Chemical Sciences, University Science Malaysia, Malay- \\ sia; ${ }^{3}$ Therapeutic Chemistry Department, National Research Centre, Cairo, Egypt
}

\begin{abstract}
Background: Dengue is one of the major public health problems in the world, affecting more than fifty million cases in tropical and subtropical region every year. The metabolome, as pathophysiological end-points, provide significant understanding of the mechanism and progression of dengue pathogenesis via changes in the metabolite profile of infected patients. Recent developments in diagnostic technologies provide metabolomics for the early detection of infectious diseases. Methods: The mid-stream urine was collected from 96 patients diagnosed with dengue fever at Penang General Hospital (PGH) and 50 healthy volunteers. Urine samples were analyzed with proton nuclear magnetic resonance ('H NMR) spectroscopy, followed by chemometric multivariate analysis. NMR signals highlighted in the orthogonal partial least square-discriminant analysis (OPLSDA) S-plots were selected and identified using Human Metabolome Database (HMDB) and Chenomx Profiler. A highly predictive model was constructed from urine profile of dengue infected patients versus healthy individuals with the total $R^{2} Y$ (cum) value 0.935 , and the total $Q^{2} Y$ (cum) value 0.832 . Results: Data showed that dengue infection is related to amino acid metabolism, tricarboxylic acid intermediates cycle and $\beta$-oxidation of fatty acids. Distinct variations in certain metabolites were recorded in infected patients including amino acids, various organic acids, betaine, valerylglycine, myoinositol and glycine. Conclusion: Metabolomics approach provides essential insight into host metabolic disturbances following dengue infection.
\end{abstract}

Key words: metabolomics, dengue, multibiomarkers, metabolite profile, 'HNMR

Received: 28 November, 2015; revised: 05 May, 2016; accepted: 25 October, 2016; available on-line: 30 March 2017

\footnotetext{
e-mail: abdelhamidzaki@hotmail.com

Abbreviations: ${ }^{1} \mathrm{H}$ NMR, proton nuclear magnetic resonance; $\mathrm{Co} A$, Coenzyme $A ; D_{2} O$, deuterium oxide; DENV, Dengue virus; $D H F$, Dengue hemorrhagic fever; DSS, Dengue shock syndrome; ELISA, Enzyme-linked immunosorbent assay; $\mathrm{HMDB}$, human metabolome database; MVA, multivariate statistical analysis; OPLS-DA, orthogonal partial least square-discriminant analysis; $P$, probability; PCA principle component analysis; $\mathrm{PCR}$, polymerase chain reaction PGH, Penang General Hospital; $Q^{2} Y$, value is analogous to $R^{2} Y$ except it is based on the cross-validated results; $R^{2} Y$, value is equivalent to the $y$-block cumulative variance captured; SNV , standard normal variate; SPSS, statistical package for the social sciences; SSC, S-sulfocysteine; TCA, trichloroacetic acid; VIP, variable importance of projection; WHO, World Health Organization
}

\section{INTRODUCTION}

Dengue is an endemic viral disease affecting tropical and subtropical regions around the world. Dengue virus (DENV) infects more than fifty million individuals every year and the incidence is increasing (Ashley, 2011), while 3.9 billion people, in 128 countries, are at risk of infection (Brady et al., 2012). It was estimated that 500000 people with severe dengue require hospitalization each year and about $2.5 \%$ of the infected dies (WHO, 2016). In Malaysia, incidence rate is high, with the average of 125 to 150 per 100000 people annually (Ahmad et al., 2012).

The diagnosis of dengue is usually made clinically and includes many symptoms that range from mild febrile illness to severe disease. Clinical features are often nonspecific and therefore require laboratory confirmation, especially for surveillance and outbreak investigations. Virus isolation provides the most convincing evidence of infection, but facilities for culture are not always available. Detection of virus-specific RNA, by polymerase chain reaction (PCR), provides relatively accurate diagnosis but requires expensive reagents and equipment. Stringent quality control is necessary to avoid false positive results due to contamination. Serological diagnosis is widely available and can provide an alternative to virus isolation or PCR in order to support the diagnosis of dengue fever. However, serum specimens may be negative for the antibodies if collected too early as the antibodies are usually detected approximately five days after the fever onset. Morevoer, the differing antibodies response patterns between primary and secondary dengue infection underscore the need to evaluate the sensitivity and specificity of commercially available tests (Hunsperger et al., 2009). Therefore, there is a great demand for the rapid detection and differentiation of dengue virus infection in the early phase of illness in order to provide rapid clinical treatment, etiologic investigation and disease control.

Metabolomics is a growing field in recent era and enables detection and quantification of the metabolome constituents, resulting from perturbation of the biological pathway that vary according to the physiological, developmental or pathologic state of the cell (Kim \& Maruvada, 2008). These variations can be used to elucidate changes of the metabotype associated with diseaserelated biochemical reactions which is often impossible using the other omics techniques. Metabolomics profiling can be applied directly in clinical testing (van Gool et al., 2010). Consequently, metabolomics is a desirable tool for diagnosing disease since metabolites are very similar across species, and represents an easily translatable and system-wide biomarker discovery approach (van der 
Greef et al., 2006). The most common and preferable biofluids used for metabolome investigation are serum and urine (Bouatra et al., 2013).

Urine is the favored biofluid among metabolomics researchers as it provides enormous amount of information. It is sterile, easy-to-obtain in large volumes, largely free from interfering proteins or lipids and chemically complex.

In this study, metabolomics analysis of urine was suggested for identifying any disturbances of metabolic processes caused by dengue infection that can reveal a variety of health and disease traits. We applied a combination of NMR spectrometry and multivariate chemometric analysis to identify potential candidate metabolite biomarkers of dengue infection in the infected individuals.

Permission to perform this research was granted by Medical Review and Ethics Committee (MREC), Ministry of Health Malaysia (Protocol No., NMRR-10-2975392). Signed informed consent was obtained from each volunteered patient and healthy individual after a full explanation of the study was provided. All data was handled confidentially and anonymously.

\section{MATERIALS AND METHODS}

Equipment. As described in our previous article (Shahfiza et al., 2015), ${ }^{1} \mathrm{H}$ NMR spectra of the urine samples from both infected and healthy individuals were acquired with AVANCE III $500 \mathrm{MHz}$ Bruker spectrometer with $\mathrm{BBO}$ broadband probe using TSP $(\delta 0.00$ ppm) as an internal standard and $\mathrm{D}_{2} \mathrm{O}$ as the frequency lock at $300 \mathrm{~K}$.

Biological samples. Sample size was calculated using Sample Size Program (http//www.kck.usm.my/ppsg/ stats_resources.htm), while in clusion an dexclusion criteria were considered as described by Shahfiza et al., (2015).

Urine samples were collected from 96 adult male patients that were serologically confirmed with dengue infection at Penang General Hospital from February to July 2011. At the same period, urine samples were collected also from 50 healthy adult male volunteers from Bukit Mertajam, Penang, Malaysia as described by Shahfizaand coworkers (2015). All the urine samples both from the patients and healthy volunteers were collected in the morning from 09.00 to $11.00 \mathrm{am}$. Dengue disease was clinically characterized by fever accompanied by at least two of the following symptoms, headaches, retroorbital pain, myalgia, arthralgia, and rash associated with platelet number below $100000 / \mathrm{mm}^{3}$. The infection status was confirmed by standard serological tests-ELISA performed at pathology laboratory of Penang General Hospital.

Sample preparation. Urine samples were collected into urine containers and kept in a cold box prior to the processing. Samples were centrifuged at $1500 \times g$ for ten minutes to remove any cellular debris. One milliliter urine aliquots were transferred to 1.5 milliliter microtubes. The aliquots were stored at $-80^{\circ} \mathrm{C}$ prior to $\mathrm{NMR}$ spectrometry (Hicks et al., 2011). Samples were prepared for NMR as described by Shahfizaand coworkers (2015).

NMR file processing. Proton NMR spectra of urine samples from both healthy and infected individuals were recorded and subsequently subjected to chemometric multivariate analysis (Shahfiza et al., 2015).

Chemometric analysis. Chenomx software (version 5.1, Alberta, Canada) was used in this study as described by Shahfiza and coworkers (2015). The averaged signals of binned ${ }^{1} \mathrm{H}$ NMR data from each sub- sample group were subjected to Principle Component Analysis (PCA) and Orthogonal Partial Least Square-Discriminant Analysis (OPLS-DA). PCA and OPLS-DA were performed with SIMCA-P+ version 12.0.1.0 (Umetrics AB, Umeå, Sweden).

Pattern recognition (multivariate analysis). The data was statistically analyzed using both Principal Component Analysis (PCA) and Orthogonal Partial Least Square-Discriminant Analysis (OPLS-DA), with SIMCA$\mathrm{P}+$ software (Version 12.0.1.0, Umetrics, Umeå, Sweden) using Pareto scaling method. OPLS-DA S-plot was used to propose potential multibiomarkers (SIMCA-P+ Version 12.0.1.0). Different data preprocessing algorithms such as normalization and standard normal variate (SNV) were applied on dengue infected subjects versus healthy control (Version 12.0.1.0, Umetrics, Umeå, Sweden).

Univariate analysis. The statistical analysis software SPSS version 20 (IBM SPSS Statistic 20) was used in this analysis.

\section{RESULTS}

Dengue infected cases were clinically characterized according to the WHO criteria, such as fever accompanied by at least two of the following symptoms, headaches, retro-orbital pain, myalgia, arthralgia, and rash associated with the platelet number below $100000 / \mathrm{mm}^{3}$. The infection status was confirmed by standard serological testsELISA performed at pathology laboratory of Penang General Hospital (PGH).

Metabolic profiling using ${ }^{1} \mathrm{H}-\mathrm{NMR}$ urine analysis. The multivariate statistical analysis (MVA) was performed on data set obtained from ${ }^{1} \mathrm{H}$ NMR spectra of urine samples. For binned spectra, Principal Component Analysis (PCA) was initially applied for data clustering.

PCA analysis failed to reveal the clustering results between these two classes as shown in Fig. 1. Due to the inconsistency and poor separation obtained by unsupervised PCA, supervised Orthogonal Partial Least Square-Discriminant Analysis (OPLS-DA) was carried out by fitting each sample of the two classes - dengue infected patients and healthy individuals. The OPLS-DA
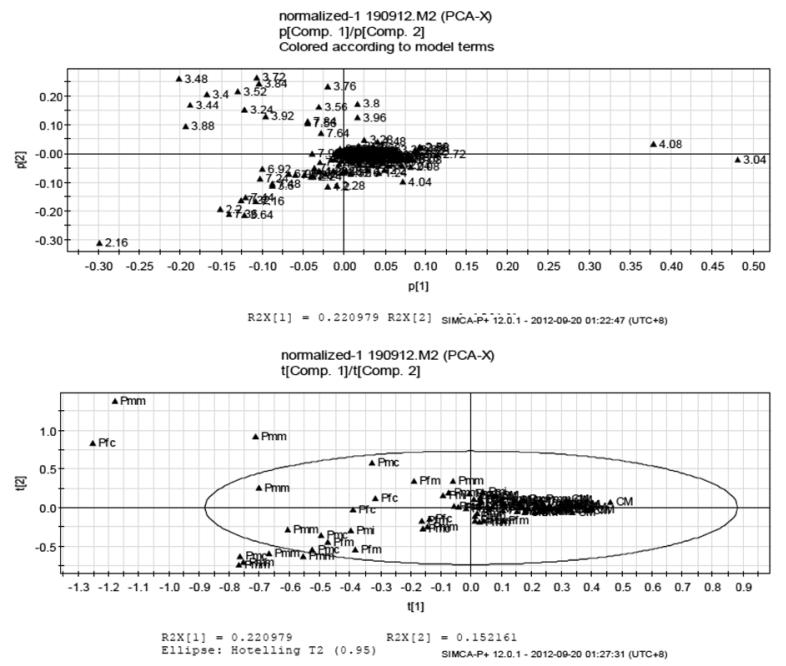

Figure 1. PCA loadings (up) and scores (below) plot showing the variation between urinary metabolic profiles of dengue-infected individuals and healthy individuals. 


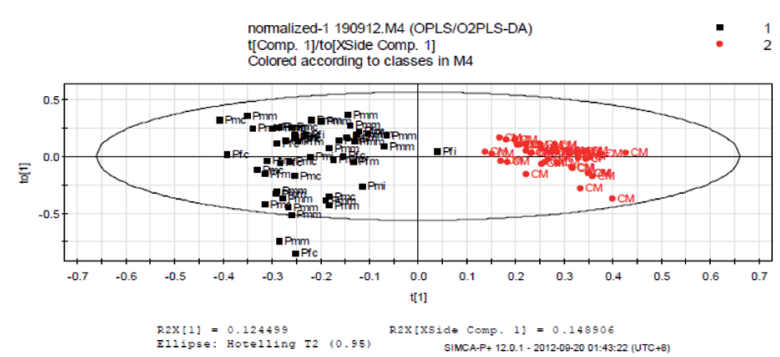

Figure 2. OPLS-DA scores plot showing the variation between urinary metabolic profiles of the dengue-infected patients and healthy individuals.

Black squares=dengue infected individuals and red circles=healthy individuals. The statistical parameters of the model were as follows $\mathrm{R}^{2} \mathrm{X}=0.9081$ and $\mathrm{Q}^{2}(\mathrm{cum})=0.7880$. The ellipse is a $95 \%$ Hotelling's $T^{2}$ ellipse.

was built to visualize the class specific segregation and to obtain the significant bins contributing to the variation between the classes, i.e. dengue infected patient versus healthy individuals. The application of this chemometric model showed a clear separation between the samples of the two classes as shown in Fig. 2.

This model showed segregation of classes with $\mathrm{R}^{2} \mathrm{X}=0.9081$ and $\mathrm{Q}^{2}$ (cum) $=0.7880$. Very narrow grouping was observed for the healthy (control) samples. Whereas, the other group classified with dengue disease was spread in the score plot which highlighted the heterogeneity of the metabolic profiles. Moreover, the spread is typical in human samples since there is enormous variation in the genetic background. In spite of these factors, the $\mathrm{Q}^{2}$ (cum) $=0.7880$ was found to be significantly high $\left(\mathrm{Q}^{2}>0.5\right)$, which implicated considerable differences in the urinary metabolic profiles of dengue patients and healthy individuals.

Visualization of metabolites using S-plot of the OPLSDA was carried out to identify potential metabolites responsible for the discrimination showed in OPLS-DA score plot. S-plot showed potentially significant metabolites based on contributions and reliability of the separation observed by OPLS-DA (Wiklund et al., 2008). Metabolites that were distant from the origin and close to

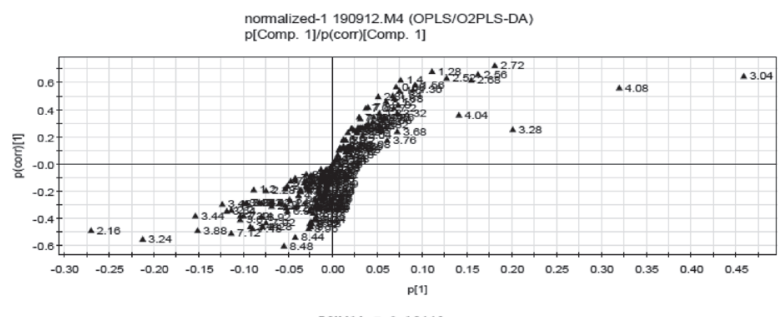

Figure 3. Representative OPLS-DA S-plot showing relative contribution of bins to clustering of dengue-infected individuals and healthy individuals.

Each point in the figure represents a bin. The $p$ (corr) [1] axis represents the correlation of the bin and the predictive variation shown in Fig. 1. The $p$ [1] axis represents the magnitude of the spectral bins. Bins with high $p$ (corr) [1] $(<-0.2$ for increased and $>0.5$ for decreased, high $p$ [1] and VIP values exceeding 1.0 as point of cut off and $p<0.05$ were selected for metabolite identification.

the vertical axis of the S-plot (Fig. 3) were responsible for the observed separation in OPLS-DA score plot (Fig. 2). Determination of those potentially influenced metabolites towards the separation in OPLS-DA models were selected based on the variable importance of projection (VIP) values exceeding 1.0 as point of cut off and have the significance of $P<0.05$ after analysis with Mann Whitney U test.

Metabolite identification. As shown in data listed in Table 1, thirteen significant spectral bins were identified from the ${ }^{1} \mathrm{H}$ NMR. The spectra contained numerous signals. The assignments were made based on the Human Metabolome Database (HMDB) and Chenomx Profiler (Chenomx NMR Suite 7.5) and on the available literature (Birungi et al., 2010). The metabolites were significantly different at $P<0.05$ after normality was assessed using Shapiro-Wilk test analysis.

Metabolites such as 4-hydroxyphenylpyruvic acid, fructose, S-sulfocysteine, acetoacetic acid, betaine and valerylglycine were found to be elevated in patients compared to healthy individuals. Meanwhile, depletion was noticed in $N$-acetylglutamic acid, creatinine, myo-inositol, creatine phosphate, succinic acid, citrate and 3-hydroxy-3methylglutarate. The discovered variation in metabolites

Table 1. OPLS-DA model bins selected for assignment of urinary metabolites influenced in the dengue infected patients compared to healthy individuals

\begin{tabular}{|c|c|c|c|}
\hline Metabolites & Chemical shift (ppm) & Differences & Significance P (Mann Whitney U Test) \\
\hline 4-Hydroxyphenylpyruvic acid & 7.12 & $\uparrow$ & $<0.001$ \\
\hline N-Acetylglutamic acid & 4.08 & $\downarrow$ & $<0.001$ \\
\hline Creatinine & 4.04 & $\downarrow$ & $<0.001$ \\
\hline Fructose & 3.88 & $\uparrow$ & $<0.001$ \\
\hline S-sulfocysteine & 3.48 & $\uparrow$ & $<0.001$ \\
\hline Acetoacetic acid & 3.44 & $\uparrow$ & $<0.001$ \\
\hline Myo-inositol & 3.28 & $\downarrow$ & $<0.006$ \\
\hline Betaine & 3.24 & $\uparrow$ & $<0.001$ \\
\hline Creatine phosphate & 3.04 & $\downarrow$ & $<0.001$ \\
\hline Succinic acid & 2.72 & $\downarrow$ & $<0.001$ \\
\hline Citrate & $2.52 ; 2.56 ; 2.68$ & $\downarrow$ & $<0.001$ \\
\hline Valerylglycine & 2.16 & $\uparrow$ & $<0.001$ \\
\hline 3-hydroxy-3-methylglutarate & 1.28 & $\downarrow$ & $<0.001$ \\
\hline
\end{tabular}

$\uparrow$ Elevation, $\downarrow$ Depletion 
could be an evident that different metabolite pathways are affected by the dengue infection.

\section{DISCUSSION}

In this study, the up- and down-regulation of certain metabolites were determined by OPLS-DA S-plot. We recorded changes in the level of thirteen metabolites identified by Human Metabolome Database (HMDB), Chenomx Profiler (Chenomx NMR Suite 7.5).

It was observed that there was an increased level of acetoacetic acid in the dengue infected patients compared to healthy individuals. The increment of acetoacetic acid level observed in this study was in agreement with the research performed by Birungiand coworkers (2010). They discovered an increased level of acetoacetic acid in samples infected with DEN-3 resulting from elevated level of amino acid, phenylalanine in the sample. Moreover, the obtained results were consistent with the increase of 4-hydroxyphenylpyruvic acid level in the urinary samples of patients infected with dengue disease. 4-hydroxyphenylpyruvic acid is an intermediate in the metabolism of the amino acid phenylalanine. Therefore it could be predicted that phenylalanine metabolism was perturbed due to the dengue infection. The elevated level of 4-hydroxyphenyl pyruvic acid could cause the increase in phenylalanine level as well. This finding was greatly supported by the classical studies of Wannemacher and coworkers (1976), regarding the plasma amino acid levels during infectious disease. Other investigation showed that the increase during infection was related to the release of phenylalanine from muscle tissues which exceeded the utilization by other cells of the viscera (Wannemacher et al., 1976). This finding explains the myalgia clinical feature of dengue disease. Furthermore, in a case study by Solomon and coworkers (2000), the progress from the classical dengue to severe dengue (DHF/DSS) was characterised by a high level of transaminases; increment in the production of phenylpyruvic acid. Thus, dengue disease seems to be another viral disease in which acetoacetic acid and phenylpyruvic acid could be good clinical indicators of the catabolic effect of the disease.

There was also an increased level of $S$-sulfocysteine metabolite in the dengue infected patients. The produced cysteine would react with endogenous sulfite thus leading to the formation of S-sulfocysteine (SSC). Elevated urinary level of SSC would cause a progressive neurologic damage. In fact, DEN-3 was associated with neurological presentation of the disease (Solomon et al., 2000).

The urine of dengue infected patient showed an increased level of valerylglycine, an acyl glycine, compared to healthy individuals. The presence of this metabolite in body fluids can be used to diagnose disorders associated with mitochondrial fatty acid $\beta$-oxidation. Therefore, it could be concluded that the impairment in fatty acid $\beta$-oxidation is associated with febrile condition.

Betaine level was also elevated in patients infected with dengue disease. This compound plays dual role either as an organic osmolyte or as a catabolic source of methyl group for use in many biochemical pathways including betaine and choline metabolism (Davies et al., 1988). Disturbances in betaine metabolism were associated with various diseases. Therefore, the urinary excretion of betaine might be a useful indicator of the development of the severe form of dengue.

The results showed an increment of urinary fructose level in dengue infected patient compared to healthy individuals. This might be due to the consumption of iso- tonic drinks by patients in order to replace fluids and electrolytes, which contains either fructose or glucose. Thus, maintenance of hydration is the major concern in the treatment of thesepatients. Thisso-called'high-fructos e'consumptioncouldacceleratethe progression of chronic kidney disease. Therefore, uncontrolled consumption of isotonic drinks could contribute to the severe dengue.

We also observed a depletion of $\mathrm{N}$-Acetylglutamic acid in the dengue infected patients. N-Acetylglutamic acid is biosynthesized from glutamic acid and acetyl-CoA by the enzyme N-Acetylglutamate synthase. N-Acetylglutamic acid acts as an activator of carbamoyl phosphate synthetase in the urea cycle. Therefore, N-Acetylglutamic synthase deficiency might lead to urea cycle failure in which ammonia conversion to urea is impossible. Urea cycle disturbance occurs in some genetic disorders and in liver failure. The results of liver failure could lead to hepatic encephalopathy due to accumulation of nitrogenous metabolites called hyperammonemia. Liver involvement in dengue infection was reported to be mild and manifested by raised liver enzymes (Martina et al., 2000). Several cases were reported where acute liver failure was the main cause of encephalopathy in patients with dengue infection (Giri et al., 2008). Moreover, acute liver failure was identified as the cause of death in DHF by dengue virus types 1 to 3 (Fadilah et al., 2000). Therefore, the presence of $\mathrm{N}$-acetylglutamic acid in the urine should be considered as a possible marker of acute liver failure in patients with dengue infection, hence a suitable treatment could be given appropriately.

There was a depletion of creatinine and creatine phosphate in the urine samples of dengue infected patients in comparison to healthy individuals. Creatinine is a breakdown product of creatine phosphate and is eliminated from the body by renal excretion at a relative constant rate. The depletion of urine creatinine, might cause a raise in its blood level. Therefore, creatinine levels in urine may be used to calculate the creatine clearance, which reflects the glomerular filtration rate; measurement of renal dysfunction. The decreased level of creatinine and creatine phosphate observed might be due to the effect of time of sample collection. The samples were collected on the fifth to seventh day after the fever onset (once the patients were confirmed with dengue infection with serological test performed by the hospital), whereas creatine (produced by creatine kinase in creatine, creatine phosphate reaction) should increase on the fourth and fifth day, diminish slowly on the following day and significantly decline on the eighth day (Agrawal et al., 1978).

There was a decreased level of myo-inositol observed in the urine samples of dengue- infected patients. Free myo-inositol (inositol) is an isomer of glucose which is synthesized in glucose-6-phosphate pathway in the kidneys. However, due to the biological significance of inositol, it was well documented that human brain contains high level of inositol and its abnormal level in this organ could contribute to variety of serious human diseases (Wang et al., 2011). Myo-inositol was suggested to play an important role in the etiology of diabetes mellitus. In diabetes complications, increased glucose levels are associated with high sorbitol accumulation in the kidney, retina, nerve, and lens, which is subsequently followed by myo-inositol depletion (Raccah et al., 1998). Diabetes mellitus is, in fact, a co-morbidity risk factor for development of DHF (Pang et al., 2012). Hence, it is important in the future to investigate whether depletion of inositol plays a role in the development of DHF as it may facilitate a better understanding of the relation be- 
tween the risk factors for DHF and the pathophysiology of DHF as well.

Decreased levels of succinic acid and 3-hydroxy3-methylglutarate were observed in the urinary samples of patients infected with dengue. The depletion of succinic acid was due to the low level of 3-hydroxy-3-methylglutarate we observed. In enzymology, 3-hydroxy-3methylglutarate serves as a substrate to succinate hydroxyl-methylglutarate CoA-transferase enzyme producing succinic acid. The elevated level of succinic acid was contributed to tetradecanedioic acid due to $\omega$-oxidation of medium chain fatty acids as a result of DEN-1 infection. However, the low level of these three compounds may occur due to the multiple reactions that utilize TCA intermediates for biosynthesis, where aspartic acid is the preferred substrate for the filling to restore the TCA levels. The TCA Cycle is a metabolic hub. Therefore, it is crucial for the cell to regulate concentrations of TCA cycle metabolites in the mitochondria; anaplerotic flux must balance cataplerotic flux in order to retain homeostasis of the cellular metabolism.

The results also showed a decrease in the level of citrate in dengue infected patients. It was suggested that urinary levels of citrate can change based on systemic $\mathrm{pH}$. Alkaline $\mathrm{pH}$ would increase the citrate level whereas acidic $\mathrm{pH}$ would decrease the urine citrate excretion. However, no significant difference in the urine $\mathrm{pH}$ of the patients was observed in this study. Generally, the pHs amongst these patients varied between 5-8. Increase of citrate level, TCA cycle intermediate, was previously implicated in liver and kidney diseases.

\section{CONCLUSION}

The results produced from ${ }^{1} \mathrm{H}$ NMR metabolomics approach provided essential insights into host metabolic disturbances after dengue virus infection. In general, the identified metabolites were related to mitochondrial energy metabolism, the level of metabolites relating to muscle and metabolites relating to circadian rhythms depended on the time of urine collection. Binning technique and pattern recognition procedures enabled us to identify thirteen significant metabolites from NMR signals which could be proposed as potential multibiomarkers in diagnosing dengue disease. Therefore, this study provides a proof-principle of using NMR based metabolomics technology in discriminating subjects of interest. Thus, this technique can be proposed to be used for early detection and as rapid diagnostic tool to reveal patient's susceptibilities to diseases by using modicum of urine as non-invasive sample.

\section{Acknowledgements}

The authors thank the workers of infectious disease unit at Penang General Hospital (PGH) for their technical assistance in sample collection from Dengue infected patients.

This work was financially supported by Advanced Medical and Dental Institute, University Science Malaysia - short term grant, No: RUT/1001/pkimia/855006.

\section{Disclosure of potential conflict of interest}

On behalf of all authors, I declare that we have no conflict of interest.

\section{REFERENCES}

Agrawal DK, Tandon P, Chaturvedi UC, Kumar A (1978) Biochemical study of certain enzymes and metabolites of the carbohydrate metabolism in the skeletal muscle of the dengue virus-infected mice. $J$ Gen Virol 40: 399-408

Ahmad Nizal MG, Rozita H, Mazrura S, Zainudin MA, Hidayatulfathi O, Faridah MA et al. (2012) Dengue infections and circulating serotypes in Negeri Sembilan, Malaysia. Malays J Pub Heal Med 12: 21-30 Ashley EA ( 2011) Dengue fever. Trends Anesth Crit Care 1: 39-41

Birungi G, Chen SM, Loy BP, Ng ML, Li SFY (2010) Metabolomics approach for investigation of effects of dengue virus infection using the EA.hy926 cell line. I Prot Res 9: 6523-6634

Brady OJ, Gething PW, Bhatt S, Messina JP, Brownstein JS, Hoen AG, Moyes CL, Farlow AW, Scott TW, Hay SI (2012) Refining the global spatial limits of dengue virus transmission by evidencebased consensus. PLoS Negl Trop Dis 6: e1760. doi:10.1371/journal. pntd.0001760

Bouatra S, Aziat F, Mandal R, Guo AC, Wilson MR, et al. (2013) The human urine metabolome. PLOS ONE 8: e73076. Doi: 10.1371

Davies SE, Chalmers RA, Randall EW, Iles RA (1988) Betaine metabolism in human neonates and developing rats. Clin Chim Acta 178: 241-249

Fadilah SAW, Sahrir S, Mazlam MZ, Raymond AA (2000) A comparison of the pattern of liver involvement in dengue hemorrhagic fever with classic dengue fever. Southeast Asian J Trop Med Public Health 31: 259-263

Giri S, Agarwal MP, Sharma V, Singh A (2008) Acute hepatic failure due to dengue, A case report 2008. http//www.casesjournal.com/ content $/ 1 / 1 / 204$

Hicks J, Sivakolundu S, Colson K (2011) Metabolomics guide user manual version 005. Bruker Biospin. Billerica, USA

Hunsperger EA, Yoksan S, Buchy P, Nguyen VC, Sekaran SD, Enria DA, Pelegrino JL, et al (2009) Evaluation of commercially available anti-dengue virus immunoglobulin M tests. Emerg Infect Dis 15: 436439. doi: 10.3201 /eid1503.080923

Kim YS, Maruvada P (2008) Frontiers in metabolomics for cancer research, proceedings of a National Cancer Institute workshop. Metabolomics 4: 105-113

Martina BEE, Koraka P, Osterhaus ADME (2000) Dengue virus pathogenesis, an integrated view. Clin Microbiol Rev 22: 564-581

Pang J, Salim A, Lee VJ, Hibberd ML, Chia KS, Leo YS, Lye DC (2012) Diabetes with hypertension as risk factors for adult dengue hemorrhagic fever in a predominantly dengue serotype 2 epidemic, A case control study. PLoS Negl Trop Dis 6: e1641. doi: 10.1371

Raccah D, Coste T, Cameron NE, Dufayet D, Vague P, Hohman TC (1998) Effect of the aldose reductase inhibitor tolrestat on nerve conduction velocity, NA/K ATPase activity, and polyols in red blood cells, sciatic nerve, kidney cortex, and kidney medulla of diabetic rats. I Diabetes Complications 12: 154-162

Shahfiza N, Hock T'T, Hashem H, Abdel-Hamid AZ (2015) Metabolomics for charachterization of gender differences in patients invected with dengue virus. Asian Pac J Trop Med 8: 451-456

Solomon T, Dung NM, Vaughn DW, Kneen R, Thao LTT (2000) Raengsakulrach B, Loan $\mathrm{H}$, et al. Neurological manifestations of dengue infection. Lancet 355: 1053-1059

van der Greef J, Hankemeier T, McBurney RN (2006) Metabolomicsbased system. biology and personalized medicine, Moving towards $\mathrm{n}=1$ clinical trials? Pharmacogenomics 7: 1087-1094

van Gool AJ, Henry B, Sprengers ED (2010) From biomarker strategies to biomarker activities and back. Drug Discov Tod 15: 121-126.

Wang Y, Liu Tb, Delmas G, Park S, Perlin D, Xue C (2011) Two major inositol transporters and their role in cryptococcal virulence. Eukaryot Cell 10: 618-628

Wannemacher RW, Klainer AS, Dinterman RE, Beisel WR (1976) The significance and mechanism of an increased serum phenylalaninetyrosine ratio during infection. Am J Clin Nutr 29: 997-1006

Wiklund S, Johansson E, Sjöström L, Mellerowicz EJ, Edlund U, Shockcor JP, et al. (2008) Visualization of GC/TOF-MS-based metabolomics data for identification of biochemically interesting compounds using OPLS class models. Analyt Chem 80: 115-122

World Health Organization (WHO) (2016) Dengue and severe dengue, media centre, fact sheet, updated April 2016 